\title{
Élaboration et nature du système enzymatique protéolytique d'Aeromonas hydrophila LP 50, isolé du lait, en fonction de la croissance
}

\author{
par \\ Françoise DENIS et Louise VEILLET-PONCET \\ Laboratoire de Microbiologie Alimentaire \\ E.N.S.A.I.A. - I.N.P.L., Nancy
}

\section{INTRODUCTION}

Bien que connus, sous d'autres noms depuis les dernières années du XIX ${ }^{e}$ siècle, en pathologie des Poïkilothermes, les Aeromonas, bactéries largement répandues dans la nature (eaux, sol, divers produits alimentaires), ne retiennent l'attention des chercheurs que depuis moins de 20 ans.

Les souches d'Aeromonas isolées, à l'origine ou non d'infection, élaborent enzymes et toxines extracellulaires.

L'importance et la nature de l'activité protéolytique du genre Aeromonas ont déjà fait l'objet de plusieurs études : ainsi, Aeromonas proteolytica, isolé en 1958 par Merkel et Traganza [21], puis largement étudié par Prescott et Willms, 1960 [28], Merkel et al., 1964 [22], Prescott et Wilkes, 1966 [29], Griffin et al., 1966-1970 [12, 13], Wilkes et al., 1969 [34], Litchfield et al., 1970 [19], Miller et Prescott, 1972 [24], Wilkes et al., 1973 [35], Kettner et al., 1974 [18], Foster et Hanna, 1974 [10]. Gross et Coles, en 1969 [15], décrivent une protéinase produite par Aeromonas hydrophila. Dahle, entre 1969 et 1971, porte son intérêt sur les protéinases extracellulaires produites par Aeromonas liquefaciens et Aeromonas salmonicida [3, 4, 5, 6, 7, 8], puis Cowell et al., 1973 [2], à propos d'Aeromonas liquefaciens. Shieh et Mac Lean, en 1975 [32], purifient une protéase extracellulaire à partir d'Aeromonas salmonicida.

Aussi, devant cette aptitude du genre Aeromonas à la protéolyse, nous a-t-il paru digne d'intérêt de préciser les caractères de l'activité protéolytique, mise en évidence par Veillet-Poncet sur des souches isolées de laits pasteurisés conditionnés [33] : la souche retenue pour effectuer cette étude est Aeromonas hydrophila LP 50. 
Afin d'approfondir l'étude de son équipement enzymatique, il convenait d'observer l'apparition et l'évolution des activités protéolytiques de ce micro-organisme, en fonction du développement de la culture dans le temps.

Dans le présent travail, nous déterminerons donc la croissance de cette souche bactérienne, puis, préalablement à toute purification, nous étudierons son système enzymatique protéolytique.

La caractérisation de ce système fera l'objet d'une prochaine publication.

\section{MATERIEL ET METHODES}

\section{Matériel d'étude - Conditions de culture}

La souche retenue, Aeromonas hydrophila LP 50, appartient au groupe des Aeromonas isolés des laits pasteurisés conditionnés par Veillet-Poncet en 1973 [33].

Les conditions de culture adoptées sont celles décrites antérieurement par Petitdemange, 1972 [26] et Veillet-Poncet, 1973 [33], qui déterminent les conditions optima de production du système enzymatique protéolytique d'Aeromonas hydrophila LP 50.

Nous retenons le milieu à base de Polypeptone (B.D. Mérieux, $20 \mathrm{~g}$ de la source azotée pour $1000 \mathrm{ml}$ d'eau distillée) glucosé à 1 p. 1000 , réparti sous un volume de $250 \mathrm{ml}$ en fioles Erlenmeyer de $500 \mathrm{ml}$, puis stérilisé à $120^{\circ} \mathrm{C}$, pendant $20 \mathrm{mn}$.

L'ensemencement des cultures se fait par addition de $1 \mathrm{ml}$ par fiole d'une suspension bactérienne en eau physiologique provenant d'une culture de $18 \mathrm{~h}$ sur milieu gélosé nutritif incliné à $30^{\circ} \mathrm{C}$.

L'ensemencement est de l'ordre de 4,5 à $5.10^{7}$ bactéries. Les cultures sont maintenues à $30^{\circ} \mathrm{C}$ (température optima de croissance d'Aeromonas hydrophila LP 50), sans agitation, dans un bain d'eau thermostaté.

\section{Méthodes d'étude de la croissance}

Afin de déterminer, avec le maximum de précision, le moment d'apparition du système enzymatique protéolytique d'A. hydrophila LP 50, nous utilisons trois techniques d'étude de la croissance : la numération des cellules bactériennes viables, la détermination de l'absorbance des suspensions bactériennes par mesure des densités optiques et la détermination du poids sec de matériel cellulaire.

Nous ne décrirons ici que la numération des cellules bactériennes viables, méthode la plus sensible d'estimation de la croissance bactérienne.

Les prélèvements $(1 \mathrm{ml})$ sont effectués à des temps donnés : immédiatement après la mise en culture (J 0$)$, après $2 \mathrm{~h}, 4 \mathrm{~h}, 6 \mathrm{~h}, 8 \mathrm{~h}$, 
$12 \mathrm{~h}, 24 \mathrm{~h}$ (J 1), 30 h, 35 h, 48 h (J 2), 54 h, 72 h (J 3), puis, de 24 h en 24 h, 96 h (J 4), 120 h (J 5, J 6, J 7, J 8).

Nous effectuons, à partir de chaque prélèvement, suffisamment dilué dans de la Tryptone-sel, cinq numérations successives sur milieu Tryptone-Agar de la FIL. L'incubation, à l'étuve, est de 48 h, à la température de $30^{\circ} \mathrm{C}$. Six essais sont réalisés, pour que les résultats obtenus soient significatifs.

\section{Obtention des extraits enzymatiques bruts}

En fonction de la courbe de croissance d'A. hydrophila LP 50, nous sélectionnons six temps d'incubation, nous permettant de suivre l'élaboration de son système enzymatique protéolytique et de l'identifier : 12 h, 24 h, 36 h, 48 h, 54 h et 96 h correspondant respectivement au milieu de la phase de ralentissement (12 h), au début ( $24 \mathrm{~h})$, milieu $(36 \mathrm{~h})$ et fin $(48 \mathrm{~h})$ de la phase stationnaire, au changement de pente du début de la phase de déclin $(54 \mathrm{~h})$ et à la phase de déclin proprement dite $(96 \mathrm{~h})$.

Les cultures, dont l'inoculum, par fiole Erlenmeyer de 2 1, contenant $1 \mathrm{l}$ de milieu à la Polypeptone glucosé, est de $4 \mathrm{ml}$, sont centrifugées à $14500 \mathrm{~g}$ pendant $25 \mathrm{mn}$ à $5^{\circ} \mathrm{C}$.

Une filtration stérilisante des surnageants est effectuée sous vide à travers un filtre Seitz dont la porosité est de $0,45 \mu$.

Les protéines des filtrats acellulaires sont concentrées par précipitation au sulfate d'ammonium. Trois zones retenues : 50 p. 100, 50 à $60 \mathrm{p} .100,60$ à $70 \mathrm{p} .100$ de saturation. Notons, cependant, que le filtrat acellulaire de $96 \mathrm{~h}$, demeurant limpide au taux de 60 p. 100 , présentera les zones suivantes : 50,50 à 70,70 à 80 p. 100 de saturation.

Après maintien à $5^{\circ} \mathrm{C}$, pendant $4 \mathrm{~h}$ pour le taux de $50 \mathrm{p} .100$ et $15 \mathrm{~h}$ pour les deux derniers taux de saturation, les protéines précipitées sont recueillies par centrifugation à $30000 \mathrm{~g}$ pendant $30 \mathrm{mn}$, à $5^{\circ} \mathrm{C}$, puis remises en solution dans un minimum de tampon véronal $0,05 \mathrm{M} \mathrm{pH} 8$ et dialysées contre ce même tampon à $5^{\circ} \mathrm{C}$, pendant $96 \mathrm{~h}$, avec renouvellement toutes les $24 \mathrm{~h}$.

Les dialysats, constituant les extraits enzymatiques bruts, sont utilisés pour la détermination des activités protéolytiques.

La concentration en protéines des extraits est déterminée selon la méthode de Lowry et al. [20] avec le réactif de Folin et Ciocalteu (Merck). La sérum-albumine bovine (Merck) est employée comme protéine-étalon.

\section{Méthodes de recherche des activités protéolytiques}

\subsection{Mesure de L'activité endopeptidasique}

Nous étudions l'activité endopeptidasique sur caséine (Hammarsten-Merck) et sur hémoglobine dénaturée (U.S. Biochemical Corporation). 


\subsubsection{Sur caséine Hammarsten}

Le mélange réactionnel a la composition suivante :

- $1 \mathrm{ml}$ d'extrait enzymatique brut ou dilué en tampon véronal $0,05 \mathrm{M} \mathrm{pH} 8$;

- 0,5 ml d'une solution de $\mathrm{CaCl}_{2} 0,06 \mathrm{M}$;

- $5 \mathrm{ml}$ d'une solution de caséine Hammarsten à 1 p. 100 en tampon tris- $\mathrm{HCl} 0,2 \mathrm{M} \mathrm{pH} 8$.

Le mélange est porté, pendant $10 \mathrm{mn}$, à $45^{\circ} \mathrm{C}$ dans un bain-marie. La réaction est arrêtée par addition de $5 \mathrm{ml}$ d'une solution d'acide trichloracétique à 10 p. 100 . Après $10 \mathrm{mn}$ de contact, le mélange est filtré sur papier Whatman n $n^{\circ}$.

Les composés solubles dans les filtrats trichloracétiques sont estimés par dosage de la tyrosine avec le réactif de Folin et Ciocalteu, selon la méthode de Lowry [20], et exprimés en termes de $\mu \mathrm{g}$ équivalent Tyrosine libéré par ml d'extrait enzymatique brut, par référence à une courbe-étalon de L-Tyrosine (Serva).

La réaction est réalisée sur $1 \mathrm{ml}$ de filtrat trichloracétique auquel nous ajoutons $2 \mathrm{ml}$ d'un réactif constitué par le mélange de $\mathrm{CO}_{3} \mathrm{Na}_{2}$ à 2 p. 100 dans la soude $0,1 \mathrm{~N}, 0,5 \mathrm{ml}$ de tartrate de sodium et potassium à 2 p. 100 et $0,5 \mathrm{ml}$ d'une solution de sulfate de cuivre à 1 p. 100 .

Le mélange est laissé $10 \mathrm{mn}$ à température ambiante, puis nous ajoutons $0,1 \mathrm{ml}$ du réactif de Folin et Ciocalteu (Merck) dilué au 1/3, une agitation immédiate est suivie d'un maintien de $30 \mathrm{mn}$ à l'obscurité pour l'obtention d'un complexe coloré stable.

L'évaluation de l'intensité du développement de la coloration bleue est effectuée à $720 \mathrm{~nm}$ à l'aide d'un spectrophotomètre Beckmann Acta II.

Un blanc est réalisé pour chaque essai.

L'activité spécifique est exprimée en $\mu \mathrm{g}$ équivalent Tyrosine par $\mathrm{mg}$ de protéines d'extrait enzymatique.

\subsubsection{Sur hémoglobine dénaturée}

L'activité endopeptidasique est déterminée selon une méthode dérivée de celle d'Anson [1], utilisée par Prescott et Willms [28], Litchfield et Prescott [19], Griffin [12], Foster et Hanna [10]. Cette méthode est la suivante : $1 \mathrm{ml}$ d'extrait enzymatique brut convenablement dilué est porté à $37^{\circ} \mathrm{C}$ avec $5 \mathrm{ml}$ d'une solution d'hémoglobine dénaturée à $4 \mathrm{p}$. 100 , dans du tampon véronal $0,05 \mathrm{M} \mathrm{pH} 8$. Après $5 \mathrm{mn}$, la réaction est arrêtée par addition de $10 \mathrm{ml}$ d'acide trichloracétique à $5 \mathrm{p} .100$. Après $20 \mathrm{mn}$ de contact, le mélange est filtré sur papier Whatman $n^{\circ} 3$.

La teneur en peptides de la fraction soluble est mesurée à $280 \mathrm{~nm}$.

Un blanc est réalisé pour chaque essai.

L'unité d'activité endopeptidasique, dans les conditions données de dosage, avec l'hémoglobine dénaturée pour substrat, est arbitraire- 
ment définie comme la variation de 0,01 unité de densité optique en $1 \mathrm{mn}$.

L'activité spécifique est mesurée par le nombre d'unités d'activité endopeptidasique par mg de protéines de l'extrait enzymatique.

\subsection{MESURE DES ACTIVITÉS EXOPEPTIDASIQUES}

\subsubsection{Activité aminopeptidasique}

Les activités de type aminopeptidase sont évaluées par mesure du taux d'hydrolyse de la L-leucine-paranitroanilide (LNA) (Serva).

$0,2 \mathrm{ml}$ du substrat LNA en solution dans le méthanol $(0,2$ p. 100) et $1,3 \mathrm{ml}$ de tampon véronal $0,05 \mathrm{M}$ pH 8 sont ajoutés à $1 \mathrm{ml}$ d'extrait enzymatique brut, ou de sa dilution, dans le tampon véronal 0,05 M $\mathrm{pH}$ 8. Le mélange est porté à $37^{\circ} \mathrm{C}$ pendant $15 \mathrm{mn}$. La réaction est arrêtée par addition de $1 \mathrm{ml}$ d'acide perchlorique dilué (17,5 $\mathrm{ml}$ d'HCL $0_{4}$ à 7 p. 100 complétés à $100 \mathrm{ml}$ avec de l'eau distillée [23]).

La variation de densité optique, provoquée par la libération de la nitroaniline de couleur jaune, est mesurée à $410 \mathrm{~nm}$.

Un blanc est réalisé à chaque essai.

L'activité aminopeptidasique est exprimée en $\mu \mathrm{g}$ de nitroaniline libéré par $\mathrm{ml}$ d'extrait enzymatique brut en $1 \mathrm{mn}$, par référence à une courbe-étalon de 3 nitroaniline (Merck).

L'activité spécifique est donnée en $\mu \mathrm{g}$ de nitroaniline par mg de protéines de l'extrait enzymatique.

\subsubsection{Activité carboxypeptidasique}

Nous étudions les activités de type carboxypeptidase sur les substrats suivants : Z-Glu-Tyr (Serva), Hippuryl-L-phénylalanine (Serva) (substrats de mise en évidence d'une carboxypeptidase de type A) et $\mathrm{N} \alpha$-Hippuryl-L-arginine (Merck) (substrat de mise en évidence d'une carboxypeptidase de type B).

Pour les substrats Z-Glu-Tyr et Hippuryl-L-phénylalanine, le dosage est le suivant $[9,14]: 0,1 \mathrm{ml}$ d'extrait enzymatique brut ou de sa dilution est ajouté à $0,9 \mathrm{ml}$ de substrat $0,002 \mathrm{M}$ en tampon véronal $0,05 \mathrm{M} \mathrm{pH}$ 8. Après un temps d'action de $15 \mathrm{mn}$ à $37^{\circ} \mathrm{C}$, la réaction est arrêtée par addition de $1 \mathrm{ml}$ de ninhydrine, selon Moore et Stein [25].

Le mélange réactionnel est immédiatement placé dans un bainmarie bouillant pendant exactement $15 \mathrm{mn}$.

Ce mélange est ensuite porté dans un bain d'eau froide pendant $5 \mathrm{mn}$, puis additionné de $8 \mathrm{ml}$ d'éthanol à $50 \mathrm{p} .100$.

Après contact de $15 \mathrm{mn}$, la teneur en tyrosine ou phénylalanine libérée est mesurée par la densité optique de la solution à $570 \mathrm{~nm}$.

Un blanc est réalisé pour chaque essai. 
La méthode, utilisée pour le substrat $\mathrm{N} \alpha$-Hippuryl-L-arginine est une application de celle de Sakaguchi [31]. Le mélange réactionnel a la composition suivante : $0,1 \mathrm{ml}$ d'extrait enzymatique brut ou de sa dilution, $0,9 \mathrm{ml}$ de substrat $0,002 \mathrm{M}$ en tampon véronal $0,05 \mathrm{M} \mathrm{pH} 8$. Après action à $37^{\circ} \mathrm{C}$ pendant $15 \mathrm{mn}$, nous ajoutons $2 \mathrm{ml}$ d'une solution à 0,02 p. 100 d'hydroxy-8-quinoléïne dans de la soude $3 \mathrm{~N}$, puis $1 \mathrm{ml}$ d'une solution aqueuse de N-bromosuccinimide à 0,1 p. 100 .

La variation de densité optique, provoquée par la libération de l'arginine, est mesurée à $500 \mathrm{~nm}$.

Un blanc est réalisé pour chaque essai.

\section{RESULTATS}

\section{Croissance d'Aeromonas hydrophila LP $\mathbf{5 0}$}

Après avoir pris en considération les limites inférieures et supérieures des dénombrements des bactéries viables au cours des six essais (fig. 1), nous traçons la courbe moyenne de croissance d'A. hydrophila LP 50 au cours du temps (fig. 2).

La courbe du taux de croissance permet de faire les remarques suivantes :

- le taux constant et maximum de croissance est atteint pendant l'intervalle de temps, $2 \mathrm{~h}-6 \mathrm{~h}$, la culture se trouve en phase de croissance exponentielle. Les bactéries prolifèrent sans qu'aucun facteur ne semble limiter leur multiplication : le temps de génération est à sa durée minimale, le taux de croissance représente alors le potentiel reproductif maximum de la souche bactérienne ;

- de $6 \mathrm{~h}$ à $24 \mathrm{~h}$, le temps de génération devenant de plus en plus long, le taux de croissance diminuant, la multiplication se ralentit. Plusieurs hypothèses permettent d'expliquer cette limitation de la multiplication bactérienne : elle peut être due soit à l'épuisement de certains éléments nutritifs, soit à l'accumulation de substances toxiques issues du métabolisme bactérien, soit à un équilibre ionique défavorable ;

- à partir de $24 \mathrm{~h}$ et jusqu'à $48 \mathrm{~h}$, la multiplication semble s'arrêter, le nombre de bactéries viables a atteint son maximum et devient stationnaire. Le taux de croissance est nul. La culture a atteint la phase de croissance stationnaire ;

- dès 48 h, s'observe la phase de déclin. Nous pourrions considérer que le taux de croissance devient négatif, que la concentration en bactéries viables s'abaisse peu à peu du fait de l'autolyse d'une partie des bactéries dont la mort n'est plus compensée par la formation de cellules nouvelles. Cependant, nous observons, au cours de cette phase, des déviations d'ordre exponentiel de décroissance, c'està-dire une remontée du taux de croissance (de $-0,13$ à 54 h à $-0,02$ 


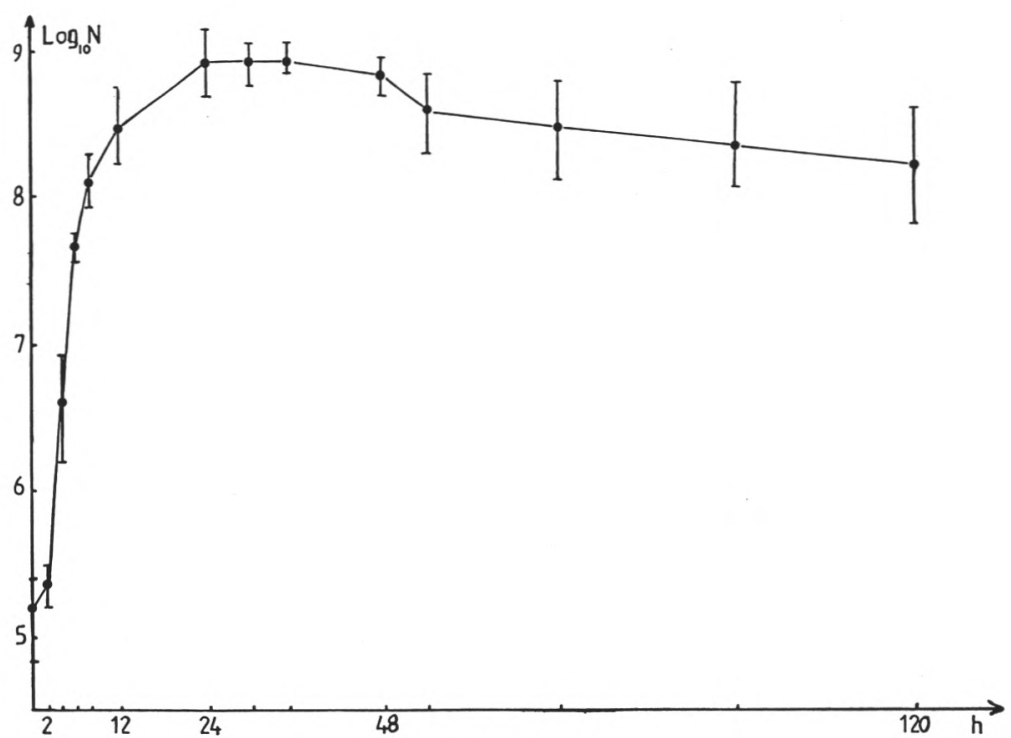

fig. 1

Croissance d'A. hydrophila LP 50 : limites inférieures et supérieures des dénombrements des bactéries viables, au cours des six essais.

$\mathrm{N}$ : Nombre de bactéries par ml.

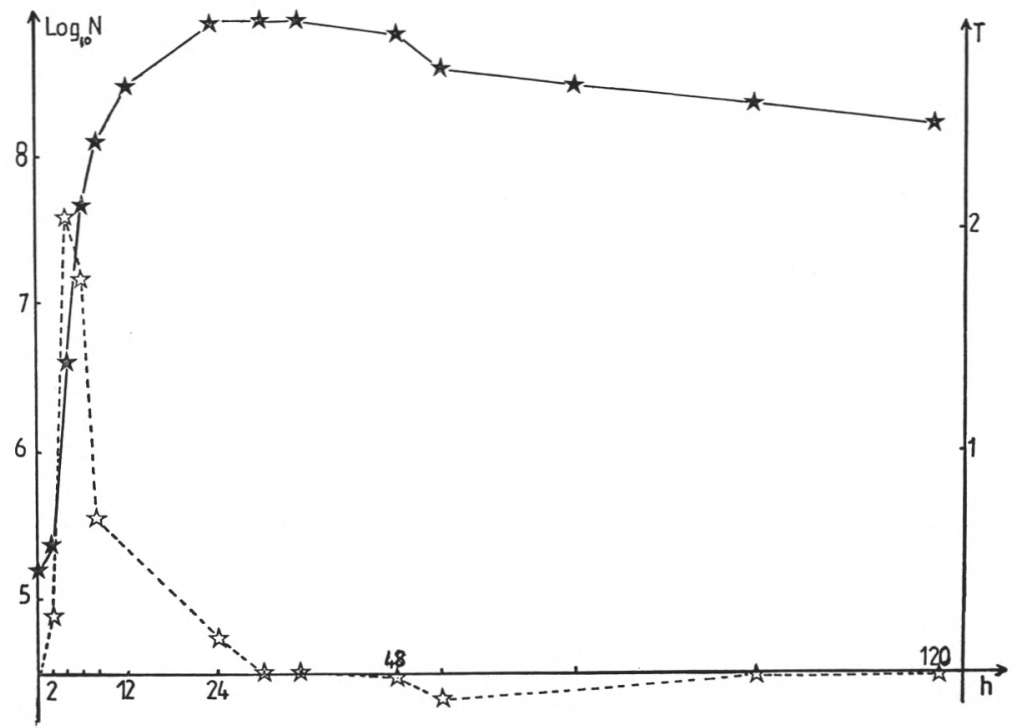

fig. 2

Courbe - Moyenne de croissance d'A. hydrophila LP 50.

$\mathrm{N}$ : Nombre de bactéries par $\mathrm{ml}$ (moyenne des six essais).

. Courbe du taux de croissance.

$\mathrm{T}$ : Nombre de divisions par heure. 
à 96 h), ce phénomène étant attribué à une reprise de la croissance pour un petit nombre de cellules, aux dépens des nutriments libérés par la dégradation lente des cellules mortes.

Nous distinguerons donc, schématiquement, dans l'évolution de notre culture bactérienne :

- une phase de latence, de $0 \mathrm{~h}$ à $2 \mathrm{~h}$ après la mise en culture ; cette phase brève (la souche se trouvant en conditions optima) est marquée par une accélération progressive du taux de croissance ;

$2 \mathrm{~h}$ à $6 \mathrm{~h}$;

- une phase de mutiplication rapide, à rythme constant, de

- une phase de multiplication ralentie, de $6 \mathrm{~h}$ à $24 \mathrm{~h}$, conduisant progressivement à :

- une phase de croissance stationnaire de $24 \mathrm{~h}$ à $48 \mathrm{~h}$,

- une phase de déclin, dès $48 \mathrm{~h}$, se terminant par l'autolyse des bactéries.

\section{Mise en évidence des différentes activités protéolytiques}

A partir des essais effectués au nombre de trois, par temps d'incubation de la culture, par type d'activité protéolytique et pour chacune des concentrations en sulfate d'ammonium retenues, nous prenons en considération la moyenne des résultats et nous déterminons les différentes activités protéolytiques.

\subsection{Activité ENDOPEPTIDASIQUe}

\subsubsection{Sur caséine Hammarsten}

Dès 12 h, l'activité caséolytique est décelable (fig. 3), la fraction de protéines précipitées à 50 p. 100 de saturation en sulfate d'ammonium présente l'activité maxima. Cette activité atteint son optimum $36 \mathrm{~h}$ après la mise en culture, suivi d'une chute graduelle jusqu'à $54 \mathrm{~h}$, et remonte très légèrement à $96 \mathrm{~h}$ d'incubation.

L'activité caséolytique, encore importante au taux de 60 p. 100, est faible à 70 p. 100 : la remontée de l'activité observée pour $96 \mathrm{~h}$ s'explique par un filtrat acellulaire demeuré limpide dans la zone 50 60 p. 100 et correspond à la fraction 50 - 70 p. 100 de saturation en sulfate d'ammonium.

Les courbes d'activités spécifiques mettent en évidence également une activité maxima pour le taux de 50 p. 100 et une activité encore non négligeable à 60 p. 100 .

\subsubsection{Sur hémoglobine dénaturée}

L'activité endopeptidasique est maxima dans la zone de précipitation 50 p. 100 (fig. 4). Cette activité, détectable dès 12 h, présente son optimum à $36 \mathrm{~h}$ d'incubation, puis elle chute brusquement jusqu'à $48 \mathrm{~h}$, pour remonter progressivement jusqu'à $96 \mathrm{~h}$. 


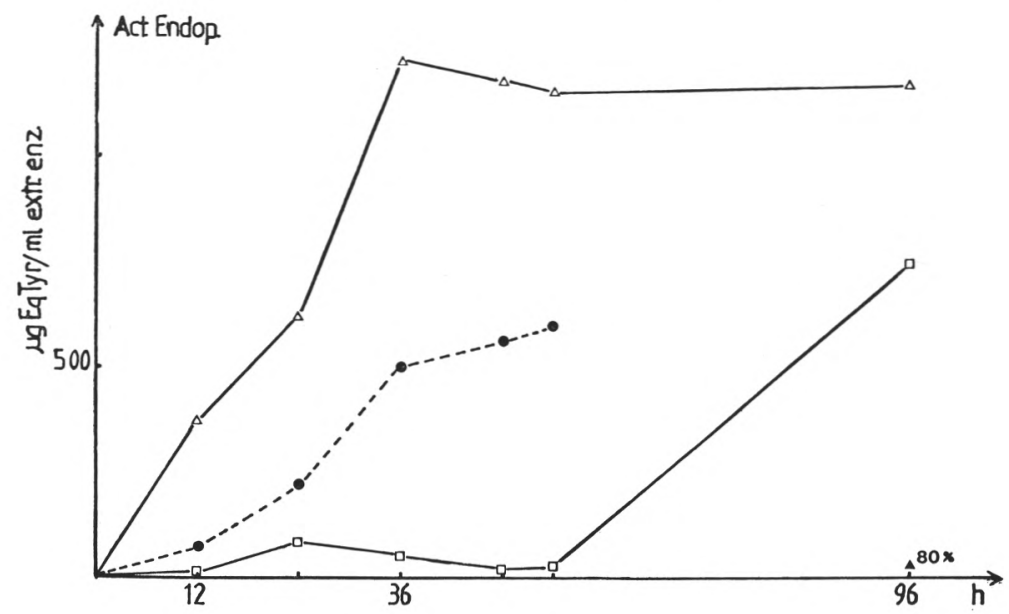

fig. 3

Activité endopeptidasique des extraits enzymatiques bruts sur caséine Hammarsten en fonction de la duree d'incubation, à différents taux de saturation en $\mathrm{SO}_{4}\left(\mathrm{NH}_{4}\right)_{2}: \triangle 50$ p. 100 . 60 p. 100, 70 p. 100.

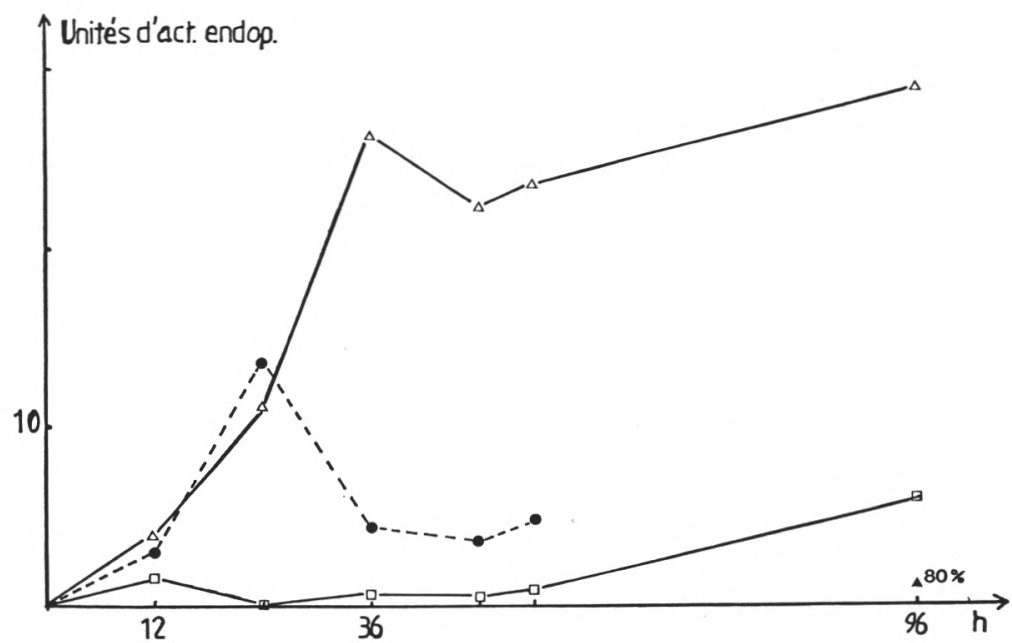

fig. 4

Activité endopeptidasique des extraits enzymatiques bruts sur hémoglobine dénaturée en fonction de la durée d'incubation, à différents taux de saturation en $\mathrm{SO}_{4}\left(\mathrm{NH}_{4}\right)_{2}: \triangle 50$ p. $100, \bullet 60$ p. 100, 70 p. 100. 
A 60 p. 100 de saturation, la courbe d'activité endopeptidasique présente un pic à $24 \mathrm{~h}$, qui est suivi d'une chute importante aux autres temps.

A 70 p. 100, l'activité est pratiquement négligeable, hormis la remontée de $96 \mathrm{~h}$, expliquée par une absence de précipitation à 60 p. 100.

Les courbes d'activités spécifiques ont sensiblement la même allure que les courbes d'activités endopeptidasiques.

\subsection{Activités EXopeptidasiques}

\subsubsection{Activité aminopeptidasique}

Nous remarquons (fig. 5) que la fraction de protéines précipitées à 50 p. 100 de saturation en sulfate d'ammonium présente, au cours du temps, la quasi-totalité de l'activité aminopeptidasique. Cette activité se trouve, en effet, presque inexistante pour les deux autres taux de précipitation 60 et $70 \mathrm{p}$. 100, hormis le temps de $24 \mathrm{~h}$ pour la courbe à 60 p. 100.

Tandis que nous notions, dès 12 , une activité conséquente pour les courbes d'activités endopeptidasiques (fig. 3 et 4), l'activité aminopeptidasique est, en revanche, extrêmement faible à ce temps d'incubation. Elle présente son optimum, là aussi, à $36 \mathrm{~h}$, diminue jusqu'à $54 \mathrm{~h}$, puis remonte vers $96 \mathrm{~h}$.

Les courbes d'activités spécifiques (fig. 6) mettent en relief l'activité aminopeptidasique contenue dans la fraction 50 p. 100, ainsi que son optimum de $36 \mathrm{~h}$, accompagné d'une chute brusque à $48 \mathrm{~h}$, qui se poursuit graduellement jusqu'à $96 \mathrm{~h}$.

\subsubsection{Activité carboxypeptidasique}

Dans nos conditions de dosage, aucune activité de type carboxypeptidasique n'a pu être mise en évidence sur les extraits enzymatiques bruts. Ceci concorde avec les études effectuées sur Aeromonas proteolytica par Griffin [12], Wilkes et al. [34], Litchfield et Prescott [19].

\section{DISCUSSION ET CONCLUSION}

Nous remarquons qu'au cours du temps les activités enzymatiques maxima sont observées dans la fraction de protéines précipitées à 50 p. 100 de saturation en sulfate d'ammonium.

Ces activités apparaissent dans le milieu de culture dès les 12 premières heures d'incubation ; nous notons cependant une activité endopeptidasique plus importante, par rapport à l'activité aminopeptidasique, tendant à montrer la synthèse et l'action préalables des endopeptidases au cours de la protéolyse. 


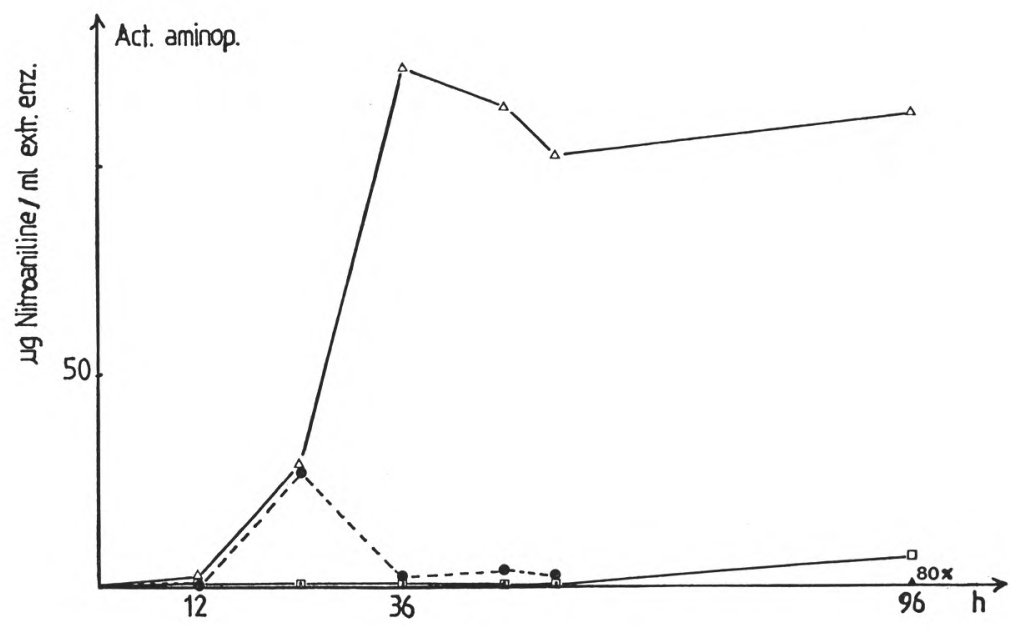

fig. 5

Activité aminopeptidasique des extraits enzymatiques bruts en fonction de la durée d'incubation, à différents taux de saturation en $\mathrm{SO}_{4}\left(\mathrm{NH}_{4}\right)_{2}$ : $\triangle 50$ p. $100, \bullet 60$ p. 100 , 口 70 p. 100.

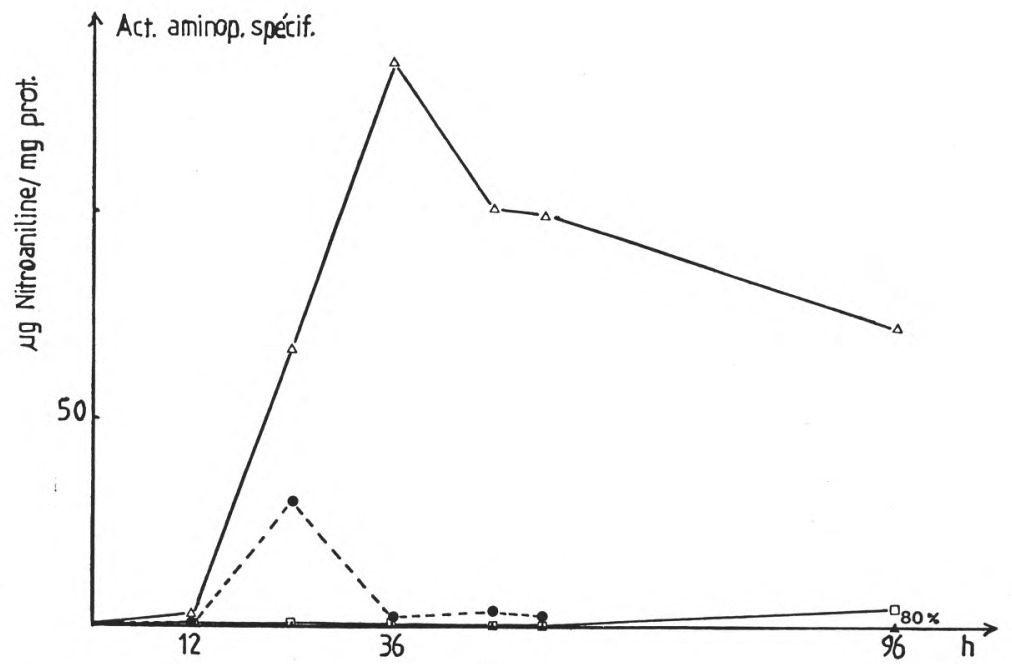

fig. 6

Activité aminopeptidasique spécifique des extraits enzymatiques bruts en fonction de la durée d'incubation, à différents taux de saturation en $\mathrm{SO}_{4}\left(\mathrm{NH}_{4}\right)_{2}: \triangle 50$ p. 100 , 60 p. 100 , 口 70 p. 100. 
Chacune des activités mises en évidence, endopeptidasique et aminopeptidasique, présente son optimum à $36 \mathrm{~h}$ d'incubation ; la production maxima du système enzymatique protéolytique semble correspondre au milieu de la phase stationnaire de la courbe de croissance.

Nous considérons que l'activité protéolytique d'A. hydrophila $L P$ 50, décelée en début de croissance, et maxima au cours de la phase stationnaire, doit être imputable en majeure partie non pas à des protéases intracellulaires libérées par autolyse, mais à des protéases de membrane, considérées comme enzymes extracellulaires.

Ceci concorderait avec les travaux de Glenn [11], Juan [16] qui associent étroitement la synthèse des exoenzymes avec la phase stationnaire de croissance. En revanche, Juffs [17], Ryden [30] estiment qu'elle suit la phase exponentielle.

Nous n'ignorons pas que le terme extracellulaire soulève de nombreuses controverses. Pollock, en 1962, définit l'enzyme extracellulaire [27]. Glenn, en 1976 [11], se référant à un grand nombre d'auteurs, établit des degrés dans la lyse cellulaire. Il distingue les vraies exoprotéines, produites principalement par les bactéries Gram posivité optimum en milieu de phase stationnaire, c'est-à-dire $36 \mathrm{~h}$ après cifiques d'un nombre important de bactéries Gram négatives, et dont la libération est limitée, dans le temps, par l'entrave que constitue la structure physico-chimique de leur paroi cellulaire.

Comme Glenn [11], nous pensons que le système protéolytique d'A. hydrophila LP 50 (bactérie Gram négative), manifestant son activité optimum en milieu de phase stationnaire, c'est-à-dire $36 \mathrm{~h}$ après la mise en culture, est constitué principalement d'exoprotéines périplasmiques. Toutefois, nous ne pouvons omettre, à ce temps de culture, la présence éventuelle d'enzymes intracellulaires libérées par lyse bactérienne. Seule, l'étude en parallèle des protéases intracellulaires libérées après broyage des cellules bactériennes et ultracentrifugation, nous permettra de lever cette incertitude.

Aeromonas hydrophila LP 50, cultivé sur milieu à la polypeptone glucosé, synthétise au cours du temps un système protéolytique exocellulaire complexe qui apparaît constitué d'au moins deux composants, endopeptidase et aminopeptidase, non isolés dans notre étude, mais mis en évidence par les activités que présentent les extraits enzymatiques obtenus à partir des filtrats acellulaires.

Ceci concorde avec les travaux de Griffin $[12,13]$, Wilkes et al. [34], Litchfield et Prescott [19] qui purifient et caractérisent chez une autre espèce, Aeromonas proteolytica, une endopeptidase et une aminopeptidase extracellulaires. 


\section{Ré s u mé}

La nature et l'évolution du système enzymatique protéolytique d'Aeromonas hydrophila LP 50, souche isolée de laits pasteurisés conditionnés, ont été étudiées en fonction du développement de la culture dans le temps.

$24 \mathrm{~h}$ après la mise en culture, sur milieu à la polypeptone glucosé, la souche atteint sa phase de croissance stationnaire, qui se prolonge jusqu'à $48 \mathrm{~h}$.

Aeromonas hydrophila LP 50 produit un système enzymatique protéolytique exocellulaire complexe doué d'activités endopeptidasique et aminopeptidasique, mais dépourvu d'activité carboxypeptidasique.

La fraction de protéines précipitées à $50 \mathrm{p} .100$ de saturation en sulfate d'ammonium renferme, au cours du temps, les activités enzymatiques maxima.

La cinétique d'apparition des deux types d'activités révèle qu'elles se manifestent dès le début de la croissance et que leur optimum se situe au milieu de la phase stationnaire.

\section{S u m m a r y}

The nature and the evolution of the proteolytic enzymatic system of Aeromonas hydrophila LP 50, strain isolated from conditioned pasteurized milks, have been studied, according to the development of the culture along time.

Twenty-four hours after the start of the culture, on glucose-polypeptone medium, the strain reaches its phase of stationary growth, which continues to forty-eight hours.

Aeromonas hydrophila LP 50 produces a complex exocellular proteolytic enzymatic system endowed with endopeptidase and aminopeptidase activities, but destituted of carboxypeptidase activity.

The fraction of proteins precipitated at 50 p. 100 of saturation in ammonium sulfate contains, in the course of time, the maxima enzymatic activities.

The cinetic of appearance of two types of activities shows that they appear immediately after the beginning of the growth and that their optimum locates itself in the middle of stationary phase. 


\section{Références bibliographiques}

[1] Anson (M. L.) (1938). - Estimation of pepsin, trypsin, papain and cathepsin with hemoglobin. J. Gen. Physiol., 22, 79-89.

[2] Cowell (J. L.), Moser (K.), Demoss (R. D.) (1973). - Tryptophanase from Aeromonas liquefaciens. Purification, molecular weight and some chemical, catalytic and immunochemical properties. Acta Biochim. Biophys., 315, 449-463.

[3] Dahle (H. K.) (1969 a). - Quantitative assay of a single proteolytic enzyme in a crude mixture of bacterial proteinases. Acta path. microbiol. Scand., $77,134-142$.

[4] Dahle (H. K.) (1969 b). - Enzymoserological separation of bacterial proteinases. Acta path. microbiol. Scand., 77, 143-148.

[5] Dahle (H. K.) (1970). - Zymograms in agar gel of some animal and bacterial proteinase. Acta path. microbiol. Scand., section B, 78, 575-578.

[6] Dahle (H. K.), SANDVIK (O.) (1971). - Comparative electrophoretic and serological analysis of Vibrio cormma and Aaeromonas liquefaciens proteinases. Acta path. microbiol. Scand., section B, 79, 686-690.

[7] Dahle (H. K.) (1971), - The purification and some properties of two Aeromonas proteinases. Acta path. microbiol. Scand., section B, 79, 726-738.

[8] Dahle (H. K.) (1971). - Regulation of the proteinase production in two strains of Aeromonas. Acta path. microbiol. Scand., section B, 79, 739-746.

[9] Desmazeaud (M. J.), Devoyod (J. J.) (1974). - Mise en évidence et caractérisation partielle de différentes peptidases chez Saccharomyces lactis. Ann. Biol. Anim. Biochim. Biophys., 14, 2, 327-341.

[10] Foster (B. G.), Hanna (M. O.) (1974). - Toxic properties of Aeromonas proteolytica. Can. J. Microb., 20, 1403-1409.

[11] GlenN (A. R.) (1976). - Production of extracellular proteins by bacteria. Ann. Rev. Microbiol., 30, 41-62.

[12] GRIFFin (T. B.) (1966). - Production, isolation and physical characterization of Aeromonas proteolytica endopeptidase. Dissert. Biochim. Nutrit., Texas $\mathrm{A}$ and $\mathrm{M}$ University, $82 \mathrm{p}$.

[13] Griffin (T. B.), Prescott (J. M.) (1970), - Some physical characteristics of a proteinase from Aeoromonas proteolytica. J. Biol. Chem., 245, 1348-1356.

[14] Gripon (J. C.), Debest (B.) (1976). - Etude électrophorétique du système protéolytique exocellulaire de Penicillium roqueforti. Le Lait, 56, 557, 423-438.

[15] Gross (R.), Coles (N. W.) (1969). - A proteinase produced by Aeromonas hydrophila. Aust. J. Sci., 31, 9, 330-331.

[16] Juan (S. M.), Cazzulo (J. J.) (1976). - The extracellular protease from Pseudomonas fluorescens. Experientia, Suisse, 32, 9, 1120-1122.

[17] Juffs (H. S.), Hayward (A. C.), Doelle (H. W.) (1968). - Growth and proteinase production in Pseudomonas $s p p$. cultivated under various conditions of temperature and nutrition. J. Dairy Res., 35, 3, 385-393.

[18] Kettner (C.), Glover (G. I.), Prescott (J. M.) (1974). - Kinetics of inhibition of Aeromonas aminopeptidase by leucine methyl ketone derivatives. Arch. Biochem. Biophys., 165, 739-743.

[19] Litchfield (C. D.), Prescott (J. M.) (1970). - Regulation of proteolytic enzyme production by Aeromonas proteolytica. I. Extracellular endopeptidase. II. Extracellular aminopeptidase. Can. J. Microbiol., 16, 1, 17-27. 
[20] Lowry (O. H.), Rosebrough (N. J.), FarR (A. L.), Randall (R. J.) (1951). Protein measurements with the folin phenol reagent. J. Biol. Chem., 193, 265-275.

[21] Merkel (J. R.), Traganza (E. D.) (1958). - Possible symbiotic role of proteolytic and cellulolytic bacteria found in the digestive system of a marine isopod. Bacteriol. Proc., p. 59.

[22] Merkel (J. R.), Traganza (E. D.), Mukherjee (B. B.), Griffin (T. B.), PresCOTT (J. M.) (1964). - Proteolytic activity and general characteristics of a marine bacterium, Aeromonas proteolytica sp. n. J. Bacteriol., 87, 1227-1233.

[23] Methods of enzymatic analysis. - Edited by Bermayer, Academic Press, New York and London, 1963, p. 830.

[24] Miller (J. M.), Prescott (J. M.) (1972). - Some immunochemical relationships of Aeromonas aminopeptidase. Int. J. Peptide Protein Res., 4, 415-419.

[25] MOORE (S.), STEIN (W. H.) (1948). - Photometric ninhydrin method for use in the chromatography of aminoacids. J. Biol. Chem., 176, 367-388.

[26] Petitdemange (E.) (1972). - Activités coagulante et protéolytique chez Aeromonas hydrophita LP 510. Thèse doctorat spécialité, Université Nancy, U.E.R. Alimentation et Nutrition.

[27] Pollock (M. R.) (1962). - Exoenzymes, in the Bacteria. Vol. IV. The physiology of growth, 121-178. Eds by Gunsalus J.C. and Stanier R., Academic Press, New York and London.

[28] PRescott (J. M.), Willms (C. R.) (1960). - Some characteristics of a proteolytic system of a marine bacterial species. Proc. Soc, Exp. Biol. Med., 103, 410-413.

[29] Prescott (J. M.), Wilkes (S. H.) (1966), - Aeromonas aminopeptidase: purification and some general properties. Arch. Biochem. Biophys., 117, 2, 328336.

[30] Ryden (A. C.), Hofsten (B. V.) (1968). - Some properties of the extracellular proteinases and the cell-bound peptidase of Serratia. Acta Chem. Scand., 22, 2803-2808.

[31] Satake (K.), Murray-Cuck (J.) (1958), - Bull. Soc. Chim. Biol., 40, 1743.

[32] SHIEH (H. S.), MAC LEAN (J. R.) (1975). - Purification and properties of an extracellular protease of Aeromonas salmonicida, the causative agent of furunculosis. Int. J. Biochem., 6, 653-656.

[33] Veillet-Poncet (L.) (1973). - La flore bactérienne indologène aéro-anaérobie des laits pasteurisés conditionnés. Cas particulier d'Aeromonas. Thèse doctorat sciences naturelles, Université Nancy, U.E.R. Alimentation et Nutrition, $n^{\circ}$ C.N.R.S. AO. 9322. Le Lait, 54, 537, 409-414 ; 538, 537-552 ; 539-540, 675-684.

[34] Wilkes (S. H.), Mukherjee (B. B.), Wagner (F. W.), Prescott (J. M.) (1969). Partial purification and some enzymatic properties of a proteinase from Aeromonas proteolytica. Proc. Soc. Exp. Biol. Med., 131, 2, 382-387.

[35] Wilkes (S. H.), Bayliss (M. E.), Prescott (J. M.) (1973). - Specificity of Aeromonas aminopeptidase toward oligopeptides and polypeptides. Eur. J. Biochem., 34, 459-466. 